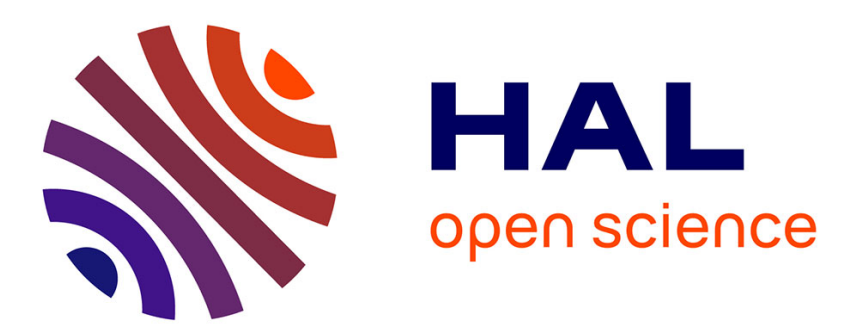

\title{
Assessment of Satellite Selection Methods under DFMC SBAS Augmentation for LPV-200 Approach Operations
}

Bouvet Denis, Alexandre Arnaudon, Rayan Ouzeri

\section{To cite this version:}

Bouvet Denis, Alexandre Arnaudon, Rayan Ouzeri. Assessment of Satellite Selection Methods under DFMC SBAS Augmentation for LPV-200 Approach Operations. ITSNT 2018, International Technical Symposium on Navigation and Timing, Oct 2018, Toulouse, France. 10.31701/itsnt2018.06 . hal01942246

\section{HAL Id: hal-01942246 \\ https://hal-enac.archives-ouvertes.fr/hal-01942246}

Submitted on 5 Dec 2018

HAL is a multi-disciplinary open access archive for the deposit and dissemination of scientific research documents, whether they are published or not. The documents may come from teaching and research institutions in France or abroad, or from public or private research centers.
L'archive ouverte pluridisciplinaire HAL, est destinée au dépôt et à la diffusion de documents scientifiques de niveau recherche, publiés ou non, émanant des établissements d'enseignement et de recherche français ou étrangers, des laboratoires publics ou privés. 


\title{
Assessment of Satellite Selection Methods under DFMC SBAS Augmentation for LPV-200 Approach Operations
}

\author{
D. Bouvet, A. Arnaudon, Thales AVS France \\ R. Ouzeri, ENAC
}

\begin{abstract}
The next generation of airborne GNSS equipment will process dual-frequency L1/L5 signals broadcast by up to four core constellations (GPS, Galileo, GLONASS and BeiDou), with integrity provided by Aircraft, Satellite or Ground Based Augmentation Systems.

The current GPS/SBAS standard RTCA/DO-229E [1] requires that airborne receivers implement a minimum of 6 channels for GPS tracking and 2 channels for SBAS tracking. For future standards addressing dual-Frequency L1/L5 multi-constellation equipment, there is a will to improve the availability figures of SBAS enabled CAT I approach, and target new operations enabled by narrower integrity bounds, by increasing the minimum number of tracking channels and possibly defining performance of the satellite selection algorithm.

On the other hand, airborne receivers have finite resources, and the "all-in-view" expectation for two or more constellations reaching 30 to 32 satellites (SV) each may not be realistic.

It is therefore necessary to define the minimum requirements for satellite selection in future DualFrequency Multi-Constellation (DFMC) standards, including the minimum number of channels to implement, or alternatively the minimal performance of the SV selection strategy.

Previous studies have already assessed a promising solution to optimize the satellite selection process under SBAS coverage: the "Downdate" method [2] [3]. The objective of this paper is to assess the performance of different selection algorithms under DFMC SBAS augmentation by taking into account not only the number of tracking channels, but also the dynamic of the reallocation process. We also consider the impact of the prediction mechanism recommended in the current GPS/SBAS standard for LPV approach.
\end{abstract}

\section{INTRODUCTION}

The next generation of EGNOS (EGNOS v3) will offer two new features:

- It will augment the Galileo positioning service (i.e. Dual Constellation capability with GPS and Galileo);
- It will provide correction data and integrity information with a second signal in the GPS L5 and Galileo E5a frequency band (i.e. Dual Frequency capability in the L1/E1 and L5/E5a frequency bands).

These features will increase the robustness of the service, and improve the performance provided to users for navigation and approach operations.

The Dual-Frequency Multi-Constellation (DFMC) SBAS MOPS under development at the EUROCAE Working Group 62 [4] aims at defining the minimum performance for future equipment processing EGNOS v3 signals, and more generally SBAS L5 signals augmenting GPS and/or Galileo constellations.

Note that future versions of the DFMC SBAS MOPS should also cover ABAS augmentation, through the Advanced RAIM capability.

To support the availability improvement offered by the single or dual-constellation SBAS L5 augmentation, we need to increase the level of minimum requirements for the airborne equipment, either through the satellite selection mechanism, or through the minimum satellite tracking capability specified in the new standards, or through both approaches.

This paper provides some elements to define these minimum requirements in the future standards.

\section{METHODOLOGY}

\subsection{Principle}

The objective is to assess for different selection methods whether the selection algorithm combined with a given maximum number of satellites tracked under "increased" constellations degrades the nominal availability performance obtained with an all-in-view receiver under nominal constellations.

For the single-constellation scenario, we consider a target of $99.8 \%$ availability for LPV-200 approaches in the LPV-200 service area (considering the extended 24+3 GPS constellation). For the dual-constellation scenario, we consider a $99.9 \%$ availability (considering nominal GPS and GAL constellations of 24 satellites each). 
For GPS, depending on the scenario, we use either the nominal constellation (24 SV), or the extended constellation (27 SV) or a $31 \mathrm{SV}$ constellation (December 2017). For Galileo, we consider either a nominal 24 SV constellation, or an increased $30 \mathrm{SV}$ constellation, assuming two additional slots per orbital plan separated by $180^{\circ}$ and centered between two nominal slots.

The figures below depict the constellations used for the performance assessment.

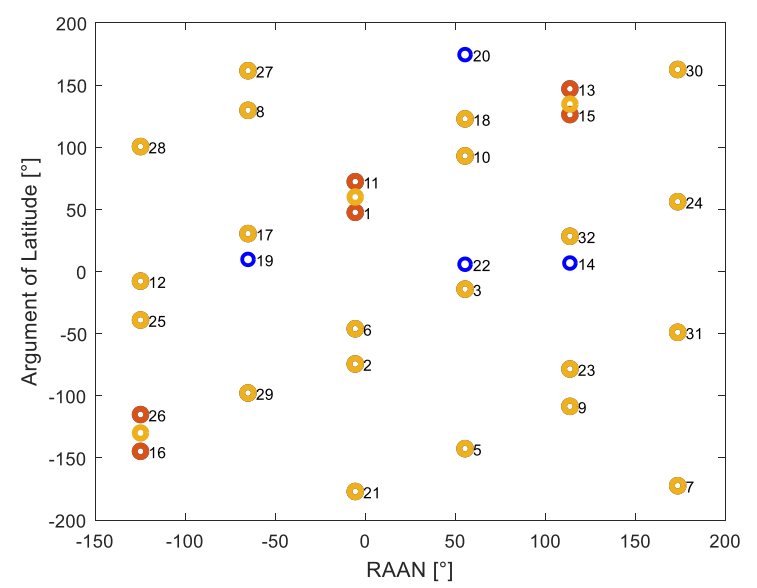

Figure 1 -GPS constellations (yellow: nominal slots, red: extended slots, blue: additional slots)

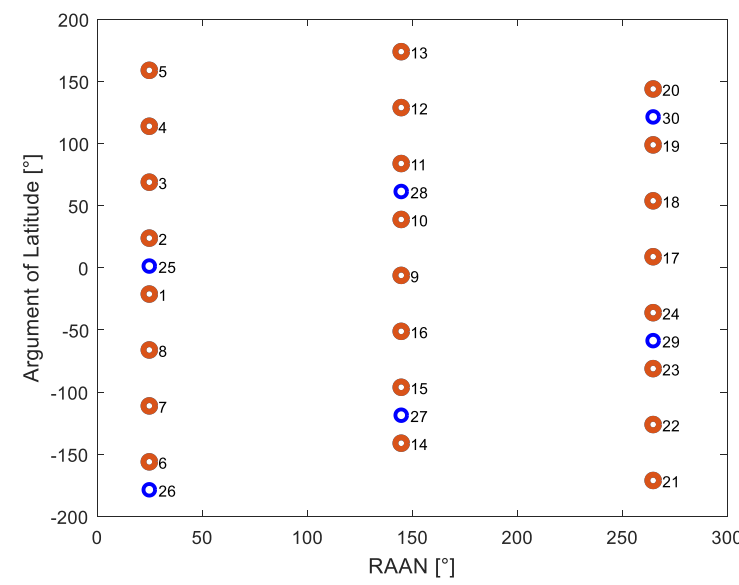

Figure 2-GAL constellation(s) (red: nominal slots, blue: additional slots)

\subsection{SBAS System Modelling}

We use an evolution of the simplified SBAS performance simulation tool MAAST published by Stanford University [5] [6] to assess SBAS L1 performance, based on the covariance matrix computation defined in [7], and on internal algorithms for the UDRE and GIVE generation.

The verification of the evolutions is not straightforward, as the exact algorithms implemented by EGNOS or WAAS systems are not known to a GNSS equipment manufacturer. Basically, it consisted in checking that the simulation results were "sufficiently" close to known references. The figures below compare the UDRE computed by the adapted version of the MAAST simulating WAAS with 38 stations to actual data broadcast by WAAS in 2015 [7].

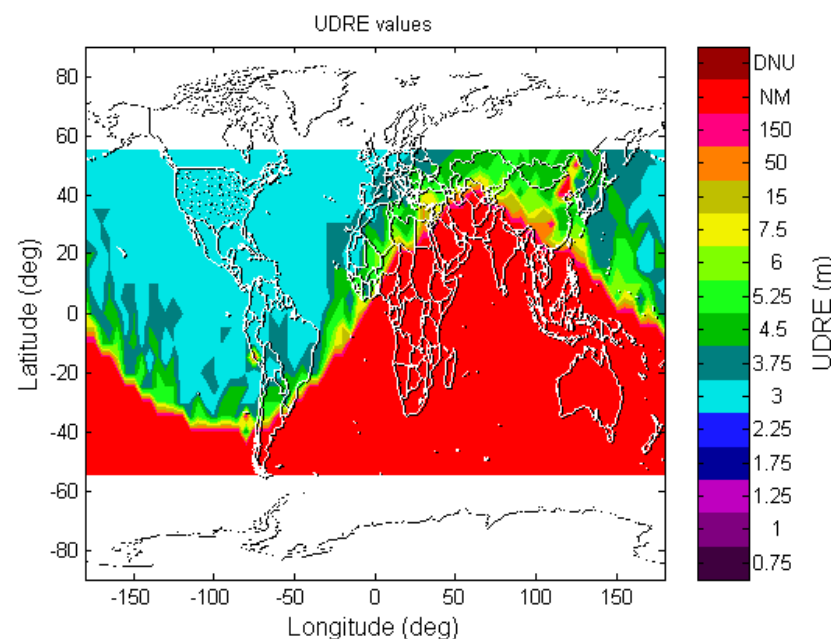

Figure 3 - Actual WAAS UDRE averaged over 24h

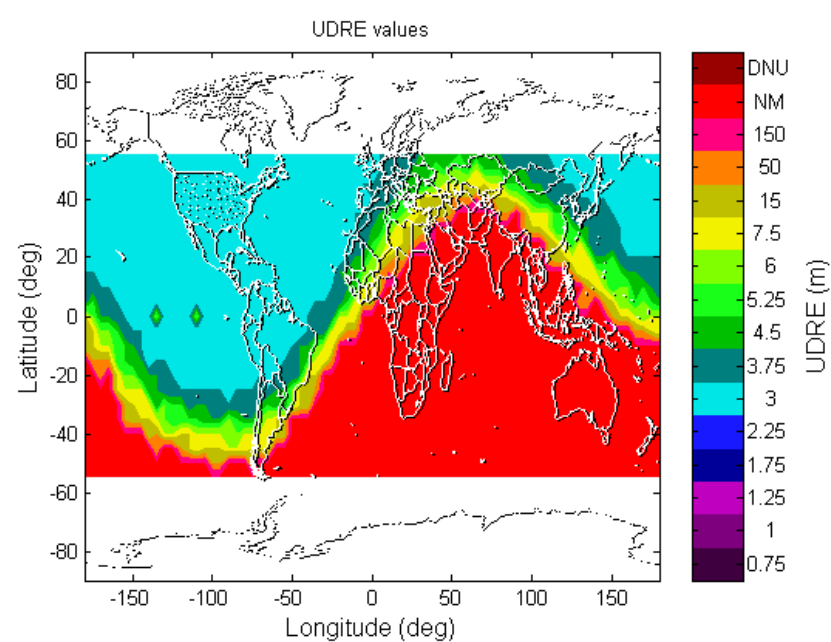

Figure 4 -UDRE computed by the adapted MAAST

Results do not match perfectly, but the overall evolutions are similar, and show that the adapted MAAST should provide representative results.

This tool has been further adapted to compute HPL and VPL issued from SBAS L5 augmentation of GPS and/or Galileo constellations, with the following evolutions:

- $\quad$ ionosphere correction model removed;

- augmentation of additional constellations implemented;

- algorithms to assess the covariance matrix and the DFRE/UDRE integrity bounds maintained, but the table defining the correspondence between DFREI and sigma_DFRE is modified to tighten the gaps between each indexed value:

Table $1-\sigma$ DFRE values used by the SBAS L5 simulator

\begin{tabular}{|c|l|c|l|}
\hline DFREI & $\sigma_{\text {DFRE }}(\mathrm{m})$ & DFREI & $\sigma_{\text {DFRE }}(\mathrm{m})$ \\
\hline 0 & 0.25 & 8 & 3.0 \\
\hline 1 & 0.50 & 9 & 3.5 \\
\hline 2 & 0.75 & 10 & 4.0 \\
\hline 3 & 1.0 & 11 & 5.0 \\
\hline 4 & 1.25 & 12 & 6.0 \\
\hline 5 & 1.5 & 13 & 8.0 \\
\hline 6 & 2.0 & 14 & 20 \\
\hline 7 & 2.5 & 15 & Do Not Use \\
\hline
\end{tabular}


In the following, simulations are run considering EGNOS with an extended monitoring network of 44 RIMS [8]:

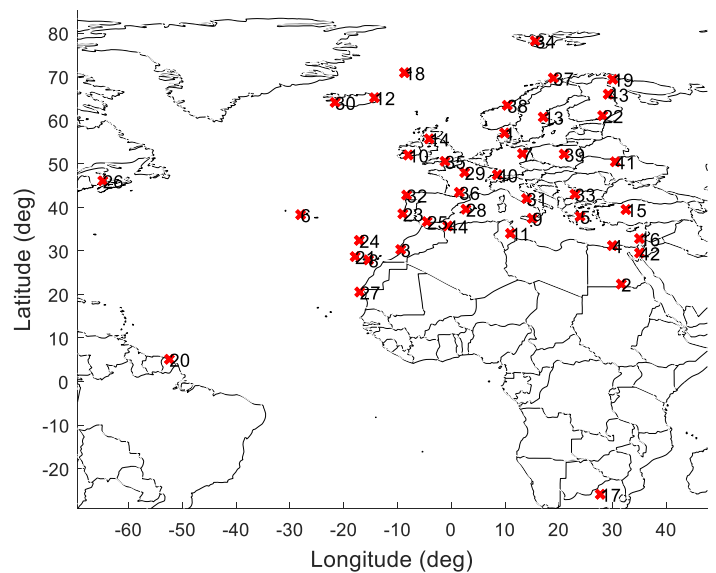

Figure 5 - Location of the 44 RIMS

In principle, EGNOS service coverage is limited in the North by 70 degrees latitude $\left(70^{\circ} \mathrm{N}\right)$, in the South by 20 degrees latitude $\left(20^{\circ} \mathrm{N}\right)$, in the East by 40 degrees longitude $\left(40^{\circ} \mathrm{E}\right)$, and in the West by 40 degrees longitude $\left(40^{\circ} \mathrm{W}\right)$. Practically, simulations are run considering a service area comprised between $30^{\circ} \mathrm{N}$ and $72^{\circ} \mathrm{N}$, and $20^{\circ} \mathrm{W}$ and $40^{\circ} \mathrm{E}$. The reason why we narrowed the area is to restrict the LPV availability analysis to the landmass region (plus ECAC islands), based on the EGNOS SoL commitment maps [9] defining the service area within which the service availability indicated on the maps is expected to be maintained under all circumstances.

As a consequence, the coverage figures provided by the adapted MAAST tool under each figure are computed by only considering the end users located over the $2^{\circ} \times 2^{\circ}$ simulation grid within the approximated boundaries of the service area delineated in Figure 6, plus northern extension.

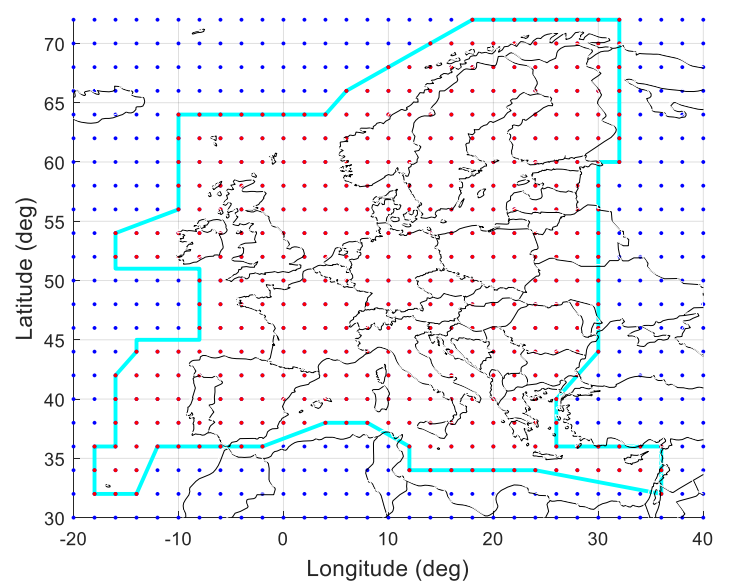

Figure 6 - Service area for LPV-200 coverage estimate

Although the results obtained with SBAS L1 are quite close to the ones observed with WAAS L1, we are aware of the fact that the adapted MAAST is not fully representative of EGNOS v3 behavior. As an illustration, figures below depict the LPV200 availability map computed with the MAAST adaptation and with ESA's
EGNOS Service Volume Simulator, using $50 \mathrm{~s}$ time steps [3]:

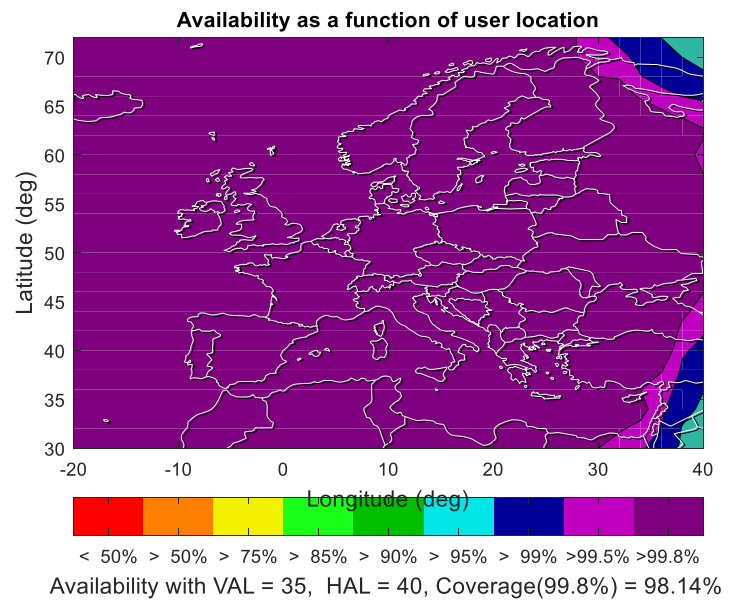

Figure 7 - LPV200 availability map obtained with the adapted MAAST assuming all-in-view dual-frequency equipment and GPS27 constellation

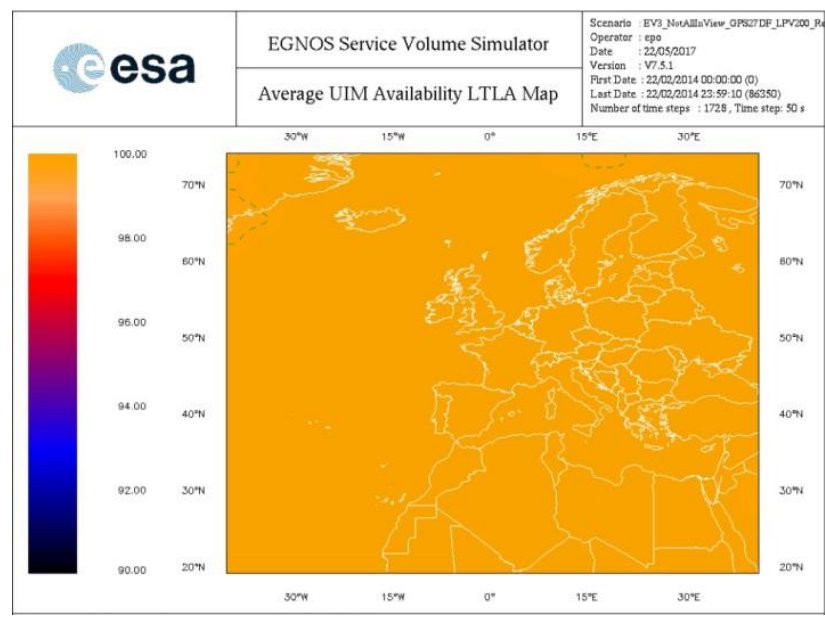

Figure 8 - LPV200 availability map obtained with ESA's simulator assuming all-in-view dual-frequency equipment and GPS27 constellation [3]

Our tool is more pessimistic in terms of predicted performance for approach operations with LPV-200 minima, as the $99.8 \%$ availability objective is met for 98.14\% of the positions within the LPV service area, versus $100 \%$ of the positions with the EGNOS Service Volume Simulator.

However, this is not seen as a major issue for the analysis carried out in this paper, as the intent is to assess the relative degradation with respect to a reference computed with the same tool: if we observe that by reducing the number of channels and/or increasing the number of visible satellites, we maintain the simulated reference performance, we will know that the simulated satellite selection algorithm is sufficient to meet the actual LPV200 performance.

\subsection{User Equipment Modelling}

Four satellite selection methods have been implemented in the adapted MAAST, with the maximum number of tracked satellites tunable. Note that the minimum considered is 10 satellites for GPS, as a minimum of 10 
L1 channels is required by DO-253D [10] for GAST-C operations.

The SV selection methods are the following:

- "Downdate" method: as defined in [2], activated every epoch;

- "Elevation" method: the $\mathrm{N}$ highest satellites are selected every epoch, where $\mathrm{N}$ is the maximum number of satellites a receiver can process;

- "Slow Elevation" method: the free channels are allocated to the highest non-tracked satellites, a channel being set free when the elevation of the tracked satellite falls below $5^{\circ}$;

- "Slow Random" method: the free channels are allocated randomly to satellites with elevation higher than $5^{\circ}$, a channel being set free when the elevation of the tracked satellite falls below $5^{\circ}$.

After the re-allocation of a channel, the satellite acquisition is modelled in two steps:

- Step 1: signal search; smoothing filter is initialized at the end of this step;

- Step 2: ephemeris collection; SV measurement are used in the PVT at the end of this period.

As the simulations are executed with a $10 \mathrm{~s}$ sampling period, $20 \mathrm{~s}$ are allocated to step 1 , and $60 \mathrm{~s}$ to step 2 . This corresponds to the $80 \mathrm{~s}$ requirement specified in DO229E [1] with a slightly different sub-allocation (14 s for search, $66 \mathrm{~s}$ for data demodulation). At first order, the approximation is deemed sufficient for GPS and for GAL satellites (for Galileo, 61 seconds are necessary to collect FNAV ephemeris).

Tropospheric and ionospheric residuals errors are modelled in accordance with the draft DFMC SBAS MOPS [4] (as a function of satellite elevation). The airborne contribution is modelled with two terms: receiver noise and multipath + antenna group delay variations. It depends on satellite elevation and on elapsed time since the initialization of the smoothing filter:

Where

$$
\sigma_{\text {air }}^{2}=2.59^{2} \times\left(\sigma_{\text {noise }}^{2}+\sigma_{M P}^{2}\right)
$$

- $\sigma_{\text {noise }}^{2}=0.15^{2} \times\left(1+\frac{2}{\alpha} \times \exp (-2 \alpha t)\right)$

- $\sigma_{M P}^{2}=\left(0.13+0.53 \times \exp \left(-e l^{\circ} / 10\right)\right) \times(1+$

$$
\left.\frac{1}{\alpha} \times \exp (-2 \alpha t)\right)
$$

- $\quad \alpha$ is defined by the smoothing filter time constant $(\alpha=0.01)$

Note that these equations model the assumptions or requirements currently considered in the DFMC SBAS MOPS, but may change in the future.

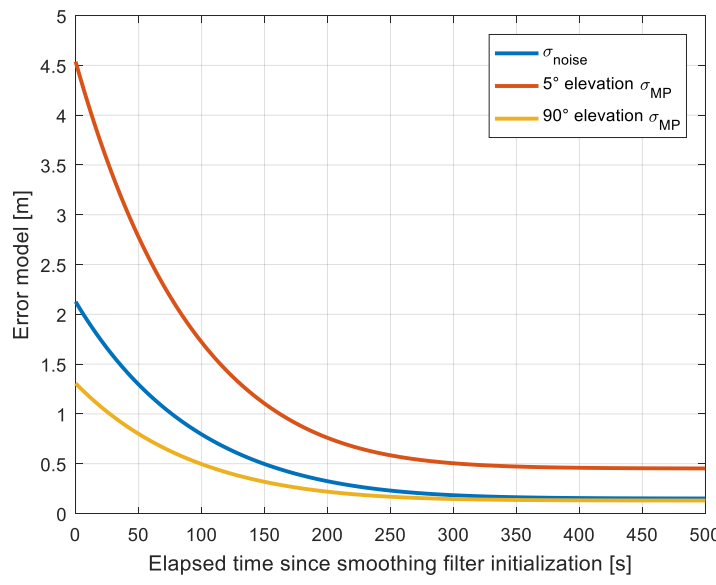

Figure 9 - Modeling of single-frequency airborne contributions

\section{COMPARISON OF THE SELECTION METHODS}

\subsection{Single Constellation Augmentation Case}

The first figure depicts the availability of the LPV200 operation assuming a 27 SV GPS constellation and an allin-view equipment. The percentage of end-users located in the service area (defined by Figure 6) with LPV-200 availability higher than $99.8 \%$ over 24 hours is $98.14 \%$ : this is the "nominal" performance to achieve. As mentioned before, the objective is to assess the degradation of performance caused by limited tracking capacities and by the SV selection algorithm.

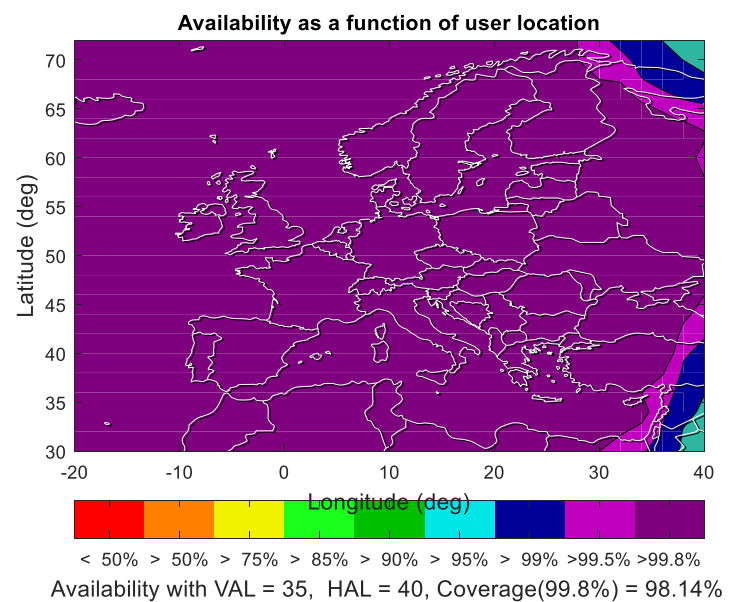

Figure 10 - LPV200 availability map with all-in-view equipment assuming GPS27 constellation

The different selection algorithms assuming a maximum of 10 tracked GPS satellites are simulated over $24 \mathrm{~h}$ considering the GPS27 constellation: the obtained $99.8 \%$ LPV200 availability coverage is strictly identical to the all-in-view equipment "nominal" performance.

When simulating a $31 \mathrm{SV}$ constellation, the selection methods have to ensure that there is no degradation with respect to the $24+3$ constellation. Figure 11 gathers the obtained availability maps. 


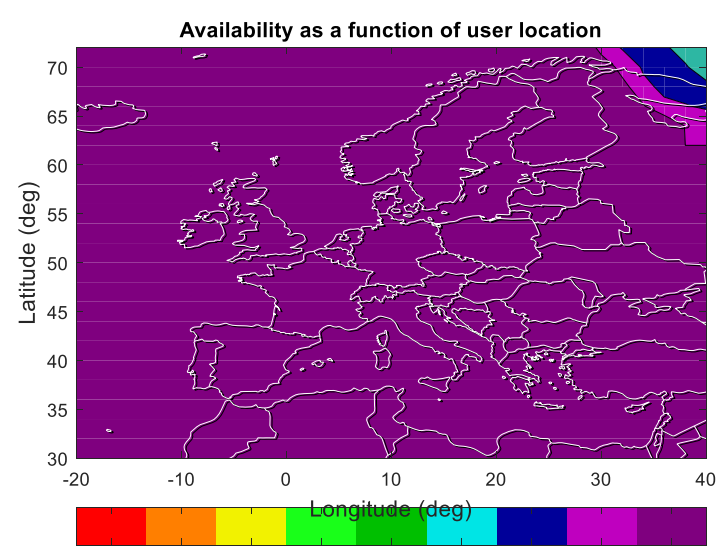

$<50 \%>50 \%>75 \%>85 \%>90 \%>95 \%>99 \%>99.5 \%>99.8 \%$ Availability with $\mathrm{VAL}=35, \mathrm{HAL}=40$, Coverage $(99.8 \%)=99.6 \%$
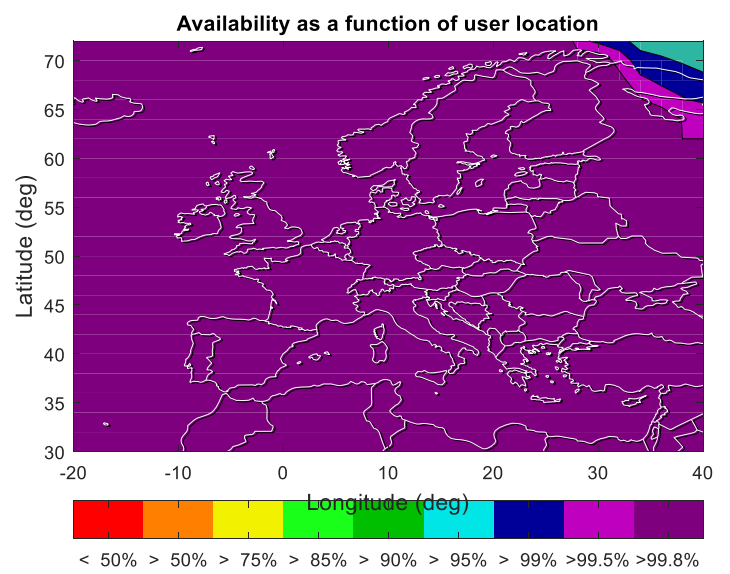
Availability with $\mathrm{VAL}=35, \mathrm{HAL}=40$, Coverage $(99.8 \%)=99.47 \%$

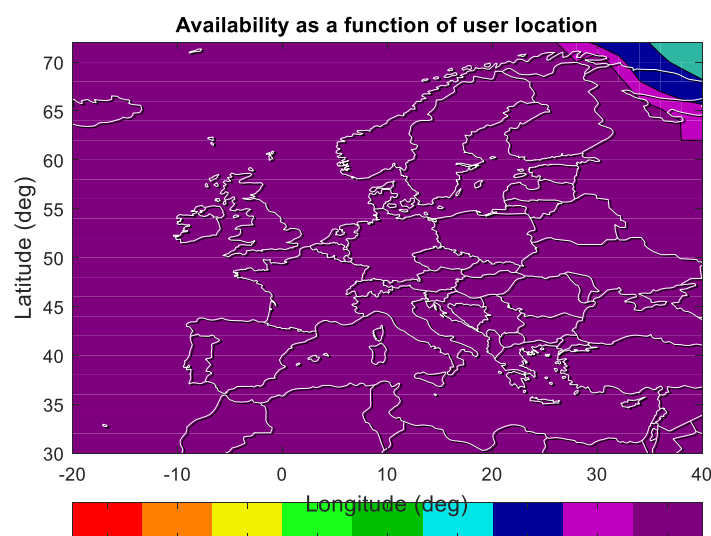

$<50 \%>50 \%>75 \%>85 \%>90 \%>95 \%>99 \%>99.5 \%>99.8 \%$ Availability with $\mathrm{VAL}=35, \mathrm{HAL}=40$, Coverage $(99.8 \%)=99.47 \%$

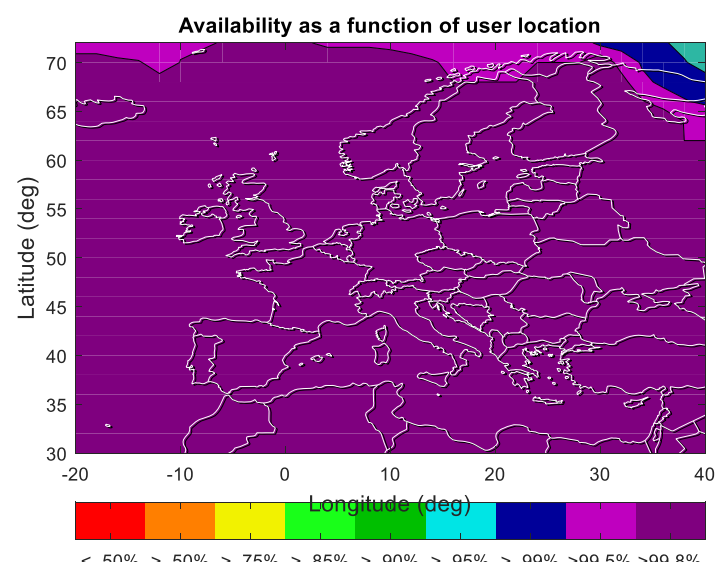

Availability with $\mathrm{VAL}=35, \mathrm{HAL}=40$, Coverage $(99.8 \%)=98.26 \%$

Figure 11 - LPV200 availability map with $10 \mathrm{SV}$ capable equipment assuming a GPS31 constellation (1: Downdate; 2: elevation; 3: slow elev.; 4: slow rand.)

With respect to the "nominal" performance, we can see that all the selection methods with a $10 \mathrm{SV}$ capable equipment have globally improved the $99.8 \%$ availability of the LPV200 approach, and that the "Downdate" method gives the best results.

We can however observe that at high latitudes, there is a local degradation of performance for the "slow random" method when compared to the reference performance obtained with the GPS27 constellation (the 99.8\% coverage is here computed by considering end users within the LPV service area whose latitude is greater than or equal to $68^{\circ} \mathrm{N}$ ).

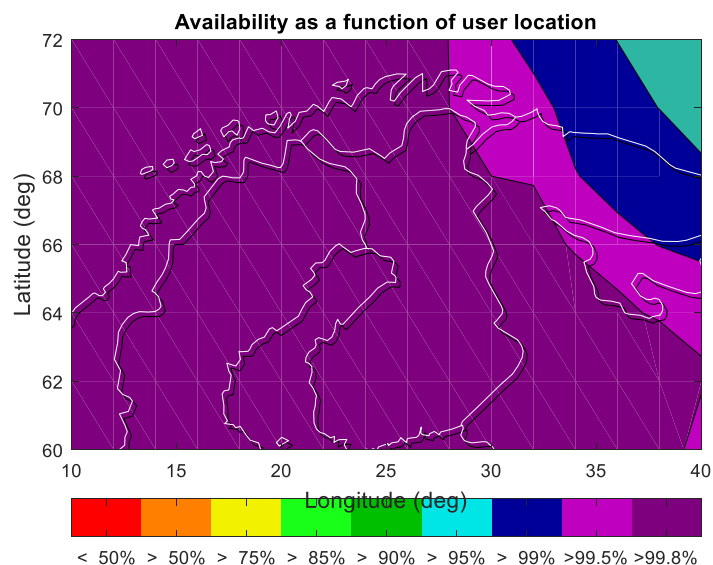

Availability with $\mathrm{VAL}=35, \mathrm{HAL}=40$, Coverage $(99.8 \%)=80.88 \%$

Figure 12 - Zoom on the LPV200 "nominal" availability map

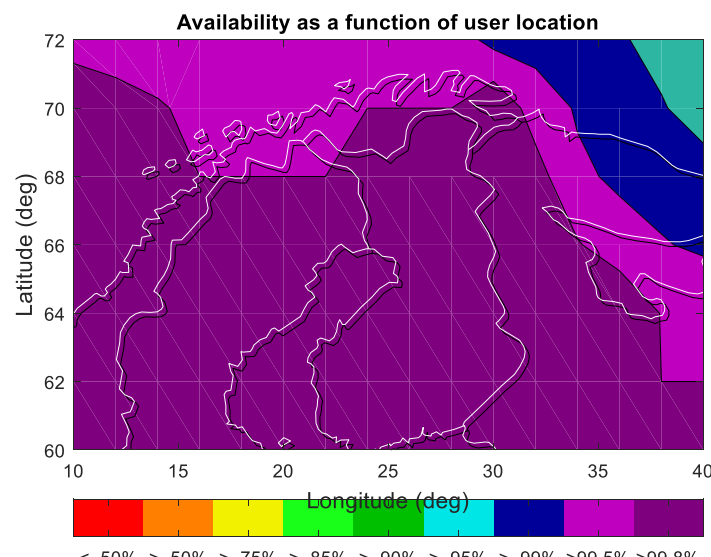

$<50 \%>50 \%>75 \%>85 \%>90 \%>95 \%>99 \%>99.5 \%>99.8 \%$ Availability with $\mathrm{VAL}=35, \mathrm{HAL}=40$, Coverage $(99.8 \%)=59.73 \%$

Figure 13 - Zoom on the LPV200 availability map with 10 SV capable equipment with "slow random" method under GPS31 constellation

For the other selection methods considering a $10 \mathrm{SV}$ tracking capability, the increased number of visible satellites improve the LPV200 availability performance, even at high latitudes.

Simulations assuming a $12 \mathrm{SV}$ capable equipment show that the selection algorithm has no impact anymore on the performance, as the $99.8 \%$ coverage of the LPV200 availability is stable over the EGNOS LPV service area. The resulting availability map is given by the following figure:

International Technical Symposium on Navigation and Timing (ITSNT) 2018 


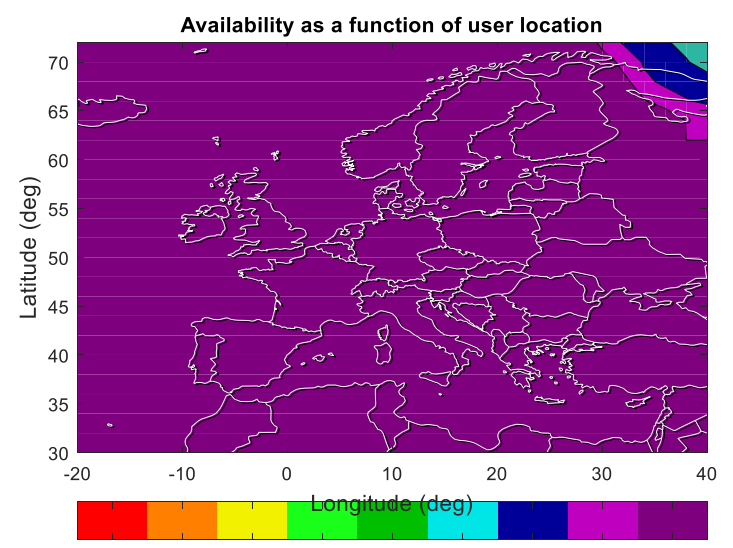

$<50 \%>50 \%>75 \%>85 \%>90 \%>95 \%>99 \%>99.5 \%>99.8 \%$ Availability with $\mathrm{VAL}=35, \mathrm{HAL}=40$, Coverage $(99.8 \%)=99.6 \%$

Figure 14 - LPV200 availability map with $12 \mathrm{SV}$ capable equipment assuming a GPS31 constellation

\subsection{Dual-constellation Augmentation Case}

The following figure depicts the "nominal" performance achieved with an all-in-view equipment using SBAS augmented satellites from GPS 24SV and GAL 24 SV constellation. Compared to the single constellation case, the objective is a $99.9 \%$ availability of the LPV approach operation.

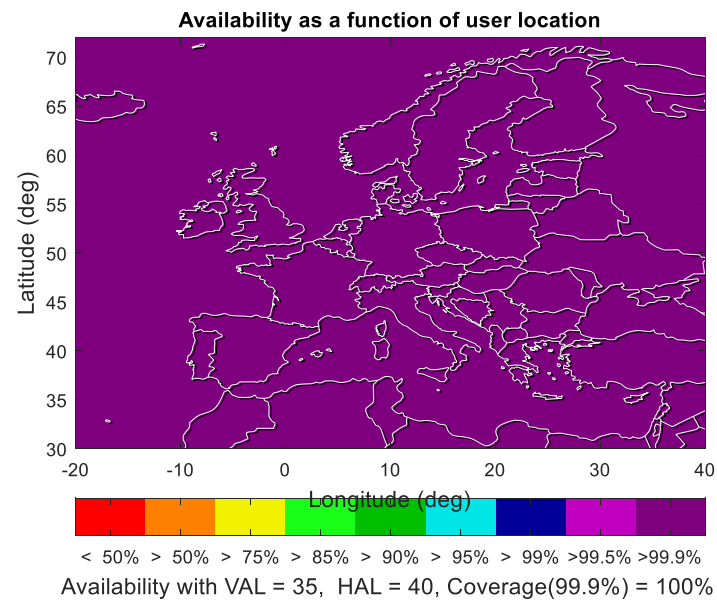

Figure 15 - LPV200 availability map with all-in-view equipment assuming GPS24+GAL24 constellation

Now assuming a 12 satellite capable equipment, and splitting equally the resources between GPS and Galileo satellites (not necessarily optimal for the "Downdate" method), we obtain the following LPV $99.9 \%$ coverage figures:

Table 2 - 12 SV capable receiver performance

\begin{tabular}{|l|l|l|l|}
\hline Selection & Trk & Constellations & Coverage \\
\hline Reference & All & GPS24+GAL24 & $\mathbf{1 0 0 \%}$ \\
\hline Downdate & 12 & GPS24+GAL24 & $100 \%$ \\
\hline Elevation & 12 & GPS24+GAL24 & $97.98 \%$ \\
\hline Slow Elevation & 12 & GPS24+GAL24 & $100 \%$ \\
\hline Slow Random & 12 & GPS24+GAL24 & $100 \%$ \\
\hline Downdate & 12 & GPS31+GAL30 & $99.19 \%$ \\
\hline Elevation & 12 & GPS31+GAL30 & $90.39 \%$ \\
\hline Slow Elevation & 12 & GPS31+GAL30 & $98.54 \%$ \\
\hline Slow Random & 12 & GPS31+GAL30 & $100 \%$ \\
\hline
\end{tabular}

For the majority of the selection algorithms, augmenting the number for available satellites degrades the performance if the equipment can only track 12 satellites. The following table gives the obtained performance with $14 \mathrm{SV}$ and $16 \mathrm{SV}$ tracking capable equipment:

Table 3 - 14 or 16 SV capable receiver performance

\begin{tabular}{|l|l|l|l|}
\hline Selection & Trk & Constellations & Coverage \\
\hline Reference & All & GPS24+GAL24 & $100 \%$ \\
\hline Downdate & 14 & GPS31+GAL30 & $100 \%$ \\
\hline Elevation & 14 & GPS31+GAL30 & $99.1 \%$ \\
\hline Slow Elevation & 14 & GPS31+GAL30 & $100 \%$ \\
\hline Slow Random & 14 & GPS31+GAL30 & $100 \%$ \\
\hline Downdate & 16 & GPS31+GAL30 & $100 \%$ \\
\hline Elevation & 16 & GPS31+GAL30 & $100 \%$ \\
\hline Slow Elevation & 16 & GPS31+GAL30 & $100 \%$ \\
\hline Slow Random & 16 & GPS31+GAL30 & $100 \%$ \\
\hline
\end{tabular}

With a 16 satellite tracking capability, the nominal coverage is obtained regardless of the satellite selection algorithm.

\section{IMPACT OF THE HPL/VPL PREDICTION MECHANISM}

\subsection{Principle}

According to DO-229E section 2.2.5.2.4 [1], Class Gamma GPS/SBAS equipment has to provide a manual or automatic means to select which type of approach will be conducted (LPV or LP, LNAV/VNAV or LNAV). This selection is based on the predicted HPL SBAS $_{\text {VPL }}$ SBAS provided by the SBAS sensor.

DO-229E defines two methods to predict the $\mathrm{HPL}_{\mathrm{SBAS}} / \mathrm{VPL}_{\mathrm{SBAS}}$, both based on the use of past VPL/VDOP and HPL/HDOP ratios and on the predicted VDOP and HDOP 5 minutes in the future (considering only SV already tracked with the exception of the SVs falling below 5 degree elevation). More precisely:

- Method \#1 multiplies the largest ratio XPL/XDOP over the previous 5 minutes by the predicted XDOP;

- Method \#2 extends the ratios collection to the previous 10 minutes, computes the largest ratio for each of the 20 consecutive intervals of $30 \mathrm{~s}$, selects the $11^{\text {th }}$ one (sorted by decreasing order) and multiplies it by the predicted XDOP.

We implemented both prediction methods in the adapted MAAST, complemented by a third method, which consists in using the current weights of satellites being tracked that are above 5 degrees 5 minutes in the future with the future lines of sight 5 minutes in the future to compute the HPL/VPL values.

The availability of the LPV-200 approach is now assessed by comparing not only the HPL and VPL to the HAL $=40$ $\mathrm{m}$ and VAL $=35 \mathrm{~m}$ values, but also the HPL and VPL predictions to the alert limits. 
If we consider the all-in-view receiver used to define the "nominal" 99.8\% availability performance when the GPS constellation has 27 satellites, the degradation of performance is shown in the following for the three methods:
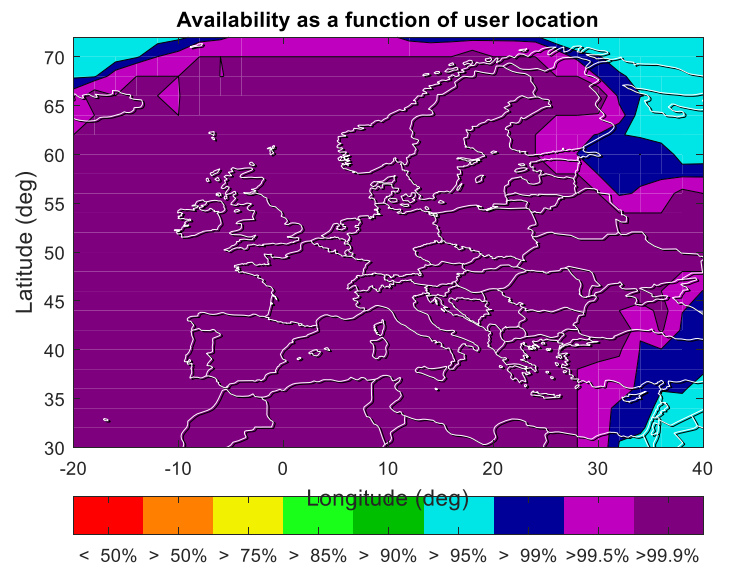
Availability with $\mathrm{VAL}=35, \mathrm{HAL}=40$, Coverage $(99.9 \%)=92.67 \%$

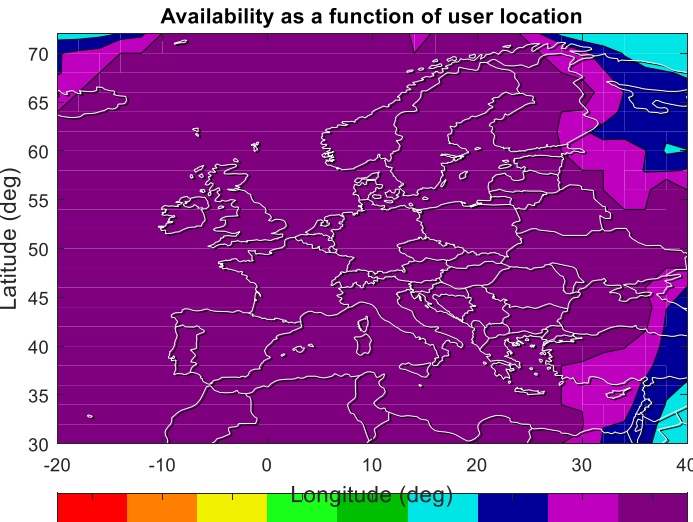

$<50 \%>50 \%>75 \%>85 \%>90 \%>95 \%>99 \%>99.5 \%>99.9 \%$ Availability with $\mathrm{VAL}=35, \mathrm{HAL}=40$, Coverage $(99.9 \%)=94.15 \%$

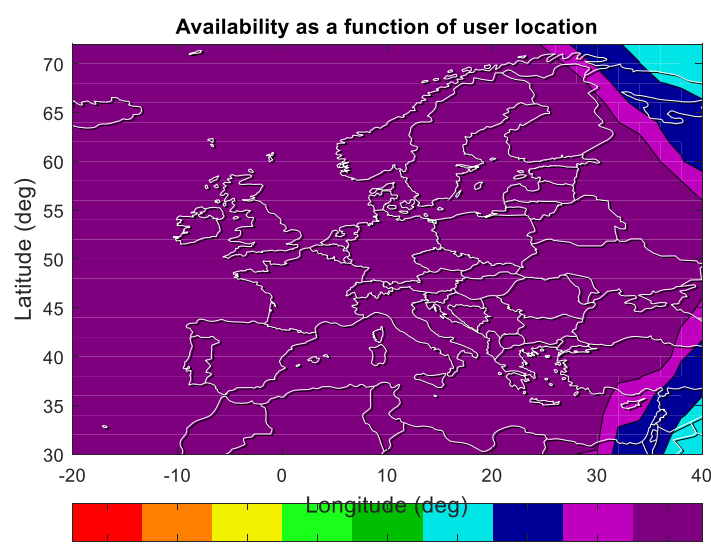

$<50 \%>50 \%>75 \%>85 \%>90 \%>95 \%>99 \%>99.5 \%>99.8 \%$ Availability with $\mathrm{VAL}=35, \mathrm{HAL}=40$, Coverage $(99.8 \%)=96.19 \%$

Figure 16 - LPV200 availability map with all-in-view equipment using the prediction capability under

GPS27 (method \#1; method \#2; method \#3)

We compared the new "nominal" performance to the results obtained with a 10 or $12 \mathrm{SV}$ capable equipment implementing one of the 4 methods of selection and one of the three methods of predictions.

Results are summarized in the following tables: for a given satellite selection method, a given satellite tracking capability and a given prediction method, a green cell International Technical Symposium on Navigation and Timing (ITSNT) 2018

13-16 Nov 2018

ENAC, Toulouse, France indicates that reference performance obtained with the allin-view equipment under nominal constellation(s) is met or exceeded assuming the same prediction method is applied.

Table 4 - Impact of the SV tracking capability Selection and Prediction methods on $99.8 \%$ coverage in single-constellation

\begin{tabular}{|l|c|c|c|c|c|c|}
\hline Selection & GPS & TRK & \multicolumn{4}{|c|}{ Prediction method } \\
\cline { 4 - 7 } & & & None & $\# 1$ & $\# 2$ & $\# 3$ \\
\hline Reference & $\mathbf{2 7}$ & All & $\mathbf{9 8 . 1 \%}$ & $\mathbf{9 2 . 9 \%}$ & $\mathbf{9 5 . 2 \%}$ & $\mathbf{9 6 . 2 \%}$ \\
\hline Downdate & 27 & 10 & $98.1 \%$ & $92.4 \%$ & $95.2 \%$ & $95.7 \%$ \\
\hline Elevation & 27 & 10 & $98.1 \%$ & $92.9 \%$ & $95.2 \%$ & $96.2 \%$ \\
\hline Slow elevation & 27 & 10 & $98.1 \%$ & $91.8 \%$ & $94.7 \%$ & $96.1 \%$ \\
\hline Slow random & 27 & 10 & $98.1 \%$ & $91.6 \%$ & $94.1 \%$ & $95.1 \%$ \\
\hline Downdate & 31 & 10 & $99.6 \%$ & $96.3 \%$ & $97.8 \%$ & $97.6 \%$ \\
\hline Elevation & 31 & 10 & $99.5 \%$ & $97.2 \%$ & $98.5 \%$ & $98.3 \%$ \\
\hline Slow elevation & 31 & 10 & $99.5 \%$ & $92.5 \%$ & $95.0 \%$ & $97.3 \%$ \\
\hline Slow random & 31 & 10 & $98.3 \%$ & $91.3 \%$ & $95.5 \%$ & $96.4 \%$ \\
\hline Downdate & 27 & 12 & $98.1 \%$ & $92.9 \%$ & $95.2 \%$ & $96.2 \%$ \\
\hline Elevation & 27 & 12 & $98.1 \%$ & $92.9 \%$ & $95.2 \%$ & $96.2 \%$ \\
\hline Slow elevation & 27 & 12 & $98.1 \%$ & $92.9 \%$ & $95.2 \%$ & $96.2 \%$ \\
\hline Slow random & 27 & 12 & $98.1 \%$ & $92.9 \%$ & $95.2 \%$ & $96.2 \%$ \\
\hline Downdate & 31 & 12 & $99.6 \%$ & $97.4 \%$ & $98.8 \%$ & $98.6 \%$ \\
\hline Elevation & 31 & 12 & $99.6 \%$ & $97.4 \%$ & $98.8 \%$ & $98.6 \%$ \\
\hline Slow elevation & 31 & 12 & $99.6 \%$ & $97.4 \%$ & $98.8 \%$ & $98.6 \%$ \\
\hline Slow random & 31 & 12 & $99.6 \%$ & $97.4 \%$ & $98.8 \%$ & $98.6 \%$ \\
\hline
\end{tabular}

We performed a similar assessment for the dualconstellation case:

Table 5 - Impact of the SV tracking capability Selection and Prediction methods on $99.9 \%$ coverage in dual-constellation

\begin{tabular}{|l|c|c|c|c|c|c|c|}
\hline Selection & GPS & GAL & TRK & \multicolumn{3}{|c|}{ Prediction method } \\
\cline { 4 - 8 } & & & & None & $\# 1$ & $\# 2$ & $\# 3$ \\
\hline Reference & $\mathbf{2 4}$ & $\mathbf{2 4}$ & All & $\mathbf{1 0 0 \%}$ & $\mathbf{1 0 0 \%}$ & $\mathbf{1 0 0 \%}$ & $\mathbf{1 0 0 \%}$ \\
\hline Downdate & 24 & 24 & 14 & $100 \%$ & $100 \%$ & $100 \%$ & $100 \%$ \\
\hline Elevation & 24 & 24 & 14 & $100 \%$ & $100 \%$ & $100 \%$ & $100 \%$ \\
\hline Slow elevation & 24 & 24 & 14 & $100 \%$ & $99.7 \%$ & $100 \%$ & $100 \%$ \\
\hline Slow random & 24 & 24 & 14 & $100 \%$ & $99.7 \%$ & $100 \%$ & $100 \%$ \\
\hline Downdate & 31 & 30 & 14 & $100 \%$ & $100 \%$ & $100 \%$ & $100 \%$ \\
\hline Elevation & 31 & 30 & 14 & $99.1 \%$ & $98.5 \%$ & $98.8 \%$ & $99.1 \%$ \\
\hline Slow elevation & 31 & 30 & 14 & $100 \%$ & $98.4 \%$ & $99.2 \%$ & $100 \%$ \\
\hline Slow random & 31 & 30 & 14 & $100 \%$ & $100 \%$ & $100 \%$ & $100 \%$ \\
\hline Downdate & 24 & 24 & 16 & $100 \%$ & $100 \%$ & $100 \%$ & $100 \%$ \\
\hline Elevation & 24 & 24 & 16 & $100 \%$ & $100 \%$ & $100 \%$ & $100 \%$ \\
\hline Slow elevation & 24 & 24 & 16 & $100 \%$ & $100 \%$ & $100 \%$ & $100 \%$ \\
\hline Slow random & 24 & 24 & 16 & $100 \%$ & $100 \%$ & $100 \%$ & $100 \%$ \\
\hline Downdate & 31 & 30 & 16 & $100 \%$ & $100 \%$ & $100 \%$ & $100 \%$ \\
\hline Elevation & 31 & 30 & 16 & $100 \%$ & $100 \%$ & $100 \%$ & $100 \%$ \\
\hline Slow elevation & 31 & 30 & 16 & $100 \%$ & $100 \%$ & $100 \%$ & $100 \%$ \\
\hline Slow random & 31 & 30 & 16 & $100 \%$ & $100 \%$ & $100 \%$ & $100 \%$ \\
\hline
\end{tabular}

The need for the HPL/VPL prediction mechanism has not been confirmed yet in the standard for DFMC SBAS equipment. But the obtained results confirm that in singleconstellation mode, having a 12 satellite tracking capability allows to guarantee the nominal performance 
when the number of usable satellites increases, regardless of the selection method implemented in the receiver.

In dual-constellation mode, having a 14 satellite tracking capability may be enough if the so-called "elevation method" is not used. However, by extending the minimum satellite tracking capability to 16 satellites, we can simplify the minimum requirements, and leave the manufacturers free to design their own satellite selection algorithm.

\section{CONCLUSION}

This paper investigated the impact of the satellite selection algorithm and the satellite tracking capability on the LPV-200 availability performance targeted by SBAS in single-constellation (GPS) and in dual-constellation (GPS + Galileo) mode.

To do so, we adapted the MAAST tool to simulate SBAS L5 augmentation and to model the behavior of DFMC SBAS equipment taking into account:

- Satellite tracking capabilities;

- Satellite selection strategies;

- Impact on the measurements of tracking channel re-allocations;

- HPL/VPL prediction mechanisms.

By comparing the "nominal" performance obtained with an all-in-view receiver under "nominal" constellation(s) to the performance obtained with DFMC SBAS receivers with finite tracking capabilities under increased constellation(s), we confirmed the good performance of the "Downdate" method, but also showed that other algorithms could give satisfying results.

As a consequence, rather than specifying the "acceptable" satellite selection algorithms to perform LPV-200 approach with SBAS L5 augmentation, we propose instead to define a minimum satellite tracking capability guaranteeing a nominal level of performance based on all in view solution, regardless of the selection method implemented by the manufacturers:

- For the single-constellation scenario, a minimum tracking capability of 12 satellites;

- For the dual-constellation scenario, a minimum tracking capability of 16 satellites (among the two augmented constellations).

In case the HPL/VPL prediction mechanism is maintained in DFMC SBAS equipment, these minimum requirements also ensure that the nominal performance is maintained.

Note that preliminary simulations performed with the same tool, targeting a 99\% coverage for "Improved SBAS CAT I" operations (with HAL $=40 \mathrm{~m}$ and VAL $=10 \mathrm{~m}$ ), showed that the minimum tracking capability of 16 satellites was not sufficient to guarantee the level of performance obtained with an all-in-view receiver under the nominal GPS24 and GAL24 constellations. Defining the minimum requirements in the DFMC SBAS MOPS for these new operations will require further analysis.

\section{ACKNOWLEDGMENTS}

This work is supported by the European GNSS Agency (GSA) through the EDG2E project (prototyping Equipment for Dual-frequency Galileo, GPS and EGNOS). However, the views expressed in this paper are those of the authors alone, and do not necessarily reflect the opinions of any other organization or person.

\section{REFERENCES}

[1] RTCA SC-159, "DO-229E, Minimum Operational Performance Standards for Global Positioning System/Satellite-Based Augmentation System Airborne Equipment, Revision E," 2017.

[2] T. Walter, J. Blanch and V. Kropp, "Satellite Selection for Multi-Constellation SBAS," Proceedings of the 29th International Technical Meeting of The Satellite Division of the Institute of Navigation (ION GNSS+ 2016), pp. 1350-1359, 2016.

[3] M. Odriozola, S. Bühler, J. Samson and S. Schlüter, "Not-all-in-view Receiver in the DFMC SBAS MOPS," in 43th meeting of the EUROCAE WG-62, Brussels (Belgium), 2017.

[4] EUROCAE WG-62, "Minimum Operational Performance Specification for Galileo / Global Positioning System / Satellite-Based Augmentation System Airborne Equipment, draft version v0.6.2," 2018.

[5] Stanford University, "MAAST, MATLAB Algorithm Availability Simulation Tool," [Online]. Available:

https://gps.stanford.edu/resources/tools/maast. [Accessed 27 August 2018].

[6] Stanford University, "Matlab Algorithm Availability Simulation Tool (MAAST) Software Developer's Guide, Version 1.1," 2002.

[7] T. Walter, A. Hansen and P. Enge, "Message Type 28," in Proceedings of the 2001 National Technical Meeting of The Institute of Navigation, Long Beach, CA, 2001.

[8] M. Tran, "Performance Evaluations of the New GPS L5 and L2 Civil (L2C) Signals," NAVIGATION, Journal of The Institute of Navigation, vol. 51, no. 3, pp. 199-212, 2004.

[9] ESA, "EGNOS V3 Phases C/D - Summary Statement of Work, Ref ESA-EGN-V3-SOW-0024, Issue 1.0," 2016.

[10] GSA, "EGNOS Safety of Life Service Definition Document, version 3.1," 2016.

[11] RTCA SC-159, "DO-253D, Minimum Operational Performance Standards for GPS Local Area Augmentation System Airborne Equipment," 2017. 